\author{
Ville Artto \\ Maija Wessman \\ Markku Nissilä \\ Erkki Säkö \\ Jarmo Liukkonen \\ Heikki Teirmaa \\ Hanna Harno \\ Hannele Havanka \\ Matti Ilmavirta \\ Aarno Palotie \\ Markus Färkkilä \\ Mikko Kallela
}

Received: 26 May 2006

Accepted in revised form: 31 August 2006

Published online: 25 October 2006

V. Artto $(\bowtie) \cdot$ H. Harno • M. Färkkilä • M.

Kallela

Department of Neurology,

Helsinki University Central Hospital,

Helsinki, Finland

e-mail: ville.artto@hus.fi

Tel.: +358-9-471-72662

Fax: +358-9-471-74003

M. Wessman • A. Palotie

The Finnish Genome Center,

Helsinki, Finland

M. Wessman

Folkhälsan Research Center,

Helsinki, Finland

M. Nissilä • E. Säkö

Turku Headache Center,

Turku, Finland

J. Liukkonen

Mikkeli Neurology Center,

Mikkeli, Finland

H. Teirmaa

Etelä-Pohjanmaa Headache Center,

Etelä-Pohjanmaa, Finland

\section{Comorbidity in Finnish migraine families}

\begin{abstract}
The objective of the study was to investigate comorbidity of migraine in Finnish migraine families. One thousand consecutive participants in the Finnish Migraine Gene Project reported their medical illnesses in addition to migraine and headache. Migraine patients $(n=678)$ reported significantly more hypotension (OR 1.43, CI 95\% 1.02-2.01), allergy (OR 1.83, CI 95\% 1.34-2.51) and psychiatric disorders (OR 4.09, CI 95\% 2.11-7.92) compared to their family members without migraine $(n=322)$. Subgroup analyses demonstrated that especially women and the group fulfilling the criteria for both migraine with and without aura were likely to have additional disorders besides their migraine. Interestingly, male migraineurs with aura reported a significant association with stroke and epilepsy. Familial migraine is comorbid with hypotension, allergy and psychiatric disorders. The association between migraine with aura and stroke and epilepsy among men of the studied families warrants further study. Clinical, pathophysiological and genetic implications of these results are discussed.
\end{abstract}

Keywords Migraine • Comorbidity • Hypotension - Psychiatric disorder • Allergy $•$ Family studies 


\section{Introduction}

Molecular genetic studies have provided important new ideas concerning migraine pathophysiology. The first two discovered migraine genes, CACNA1A and ATP1A2 [1, 2], are genes for the rare migraine subtype, familial hemiplegic migraine (FHM). CACNA1A codes for a voltagedependent, P/Q-type, alpha-1A subunit calcium channel and ATP1A2 for a $\mathrm{Na}^{+}, \mathrm{K}^{+}$-ATPase alpha-2 subunit. These findings lead to the hypothesis that migraine could be a channelopathy, something that resides in the neuronal membrane, making it more excitable [3, 4]. Similar breakthroughs are awaited for the more common migraine forms, migraine with and without aura. These disorders are likely heterogenous and harder to tackle. It seems that new angles on the migraine phenotype could facilitate the discovery of a predisposing gene variant. One aspect that might give additional information on the migraine phenotype and might also be helpful in locating new susceptibility genes is migraine comorbidity [5]. This idea is based on the hypothesis that if two diseases share common metabolic pathways and part of their pathophysiology it would be easier to locate the genes by combining information concerning the two disorders. Comorbidity of migraine has been extensively studied and comprehensive reviews have been published. An association has been reported with allergies, mitral valve prolapse, hypotension, hypertension, stroke, depression and anxiety [5]. Based on all the current data, Breslau and Rasmussen [6] underlined the co-occurrence with psychiatric conditions, epilepsy and stroke.

In Finland a large family study, the Finnish Migraine Gene Project, is ongoing [7, 8]. Large Finnish families are studied clinically and analysed with current molecular genetic methods in search for genes predisposing to common forms of migraine, especially migraine with aura. The objective of the present study is to analyse comorbidity of migraine in the Finnish migraine families and to discuss how familial comorbidities could help in the genetic studies. The chosen clinical setting, large migraine families, and the scope of the study, 1000 migraineurs, give unusual importance to the presented observations.

\section{Subjects and methods}

Study design

Consecutively identified families with at least three first-degree family members affected with migraine were recruited nationwide for the Finnish Migraine Gene Project [7]. The families were identified from among patients attending outpatient headache clinics in six Finnish cities (Helsinki, Kemi, Turku,
Jyväskylä, Mikkeli and Seinäjoki). Nine neurologists (MK, MF, MN, HH, ES, MI, JL, HT and HH) were in charge of the recruitment. Once a member of the family, the index case, was clinically diagnosed by a neurologist as suffering from migraine, the index case was asked to contact all other members of the family believed to suffer from migraine and ask if they would be willing to participate in the study. If at least three possible migraine sufferers were willing to participate, a validated questionnaire, the Finnish Migraine Specific Questionnaire for Family Studies (FMSQFS), was mailed to each of them, their parents and siblings [8]. The FMSQFs has been shown to be both specific and sensitive in diagnosing migraine in a family setting and also in differentiating migraine with aura (MwA) from migraine without aura (MwoA) [8].

All participants gave their informed consent to take part in the study, which was approved by the local ethics committee in Helsinki.

From among the first 1000 participants, belonging to 251 families, comorbidity of migraine was studied by observing medical illnesses reported by the participants in the FMSQFS. Table 1 presents the wording (translated into English) concerning these illnesses in the questionnaire. Migraine cases were compared to family members without migraine. Differences between migraine subgroups were noted as well. The FMSQFS divides participants in the following diagnostic categories: migraine aura without headache (i.e., migraine equivalents, Eqv), migraine with aura (MwA), migraine with and without aura (MwA+MwoA), migraine with aura not fulfilling the current criteria (Unc.mig), migraine without aura (MwoA), headache other than migraine (HA) and no headache (NO). All these categories are based on the current criteria of the International Headache Society [9]. The Unc.mig group refers to patients who have migraine headache according to the criteria with atypical aura symptoms that do not fulfil the criteria. All comorbidities reported by the patients were noted and the following were chosen for detailed inspection: epilepsy, stroke, hypertension, hypotension, allergy and psychiatric disorders.

\section{Statistics}

The data were recorded and analysed with a Statview 5.0 software package (Abacus Concepts, Inc., Berkley, CA, USA, 1996). Comorbidities in various study groups were analysed with multiple logistic regression. For age adjustment participants were divided into age groups $<25$, between 25 and 50, and $>50$ years of age, after which CIA Software (Trevor N. Bryant, 2000) was used to calculate age-adjusted standardised rates for different comorbidities. Multiple logistic regression odds ratios (OR) adjusted for age are shown with $95 \%$ confidence intervals.

\section{Results}

The 1000 participants belonged to 251 separate families; 606 were women, 394 men. The mean age was 40.0 years 
Table 1 Wording used in the FMSQFS concerning illnesses of participants

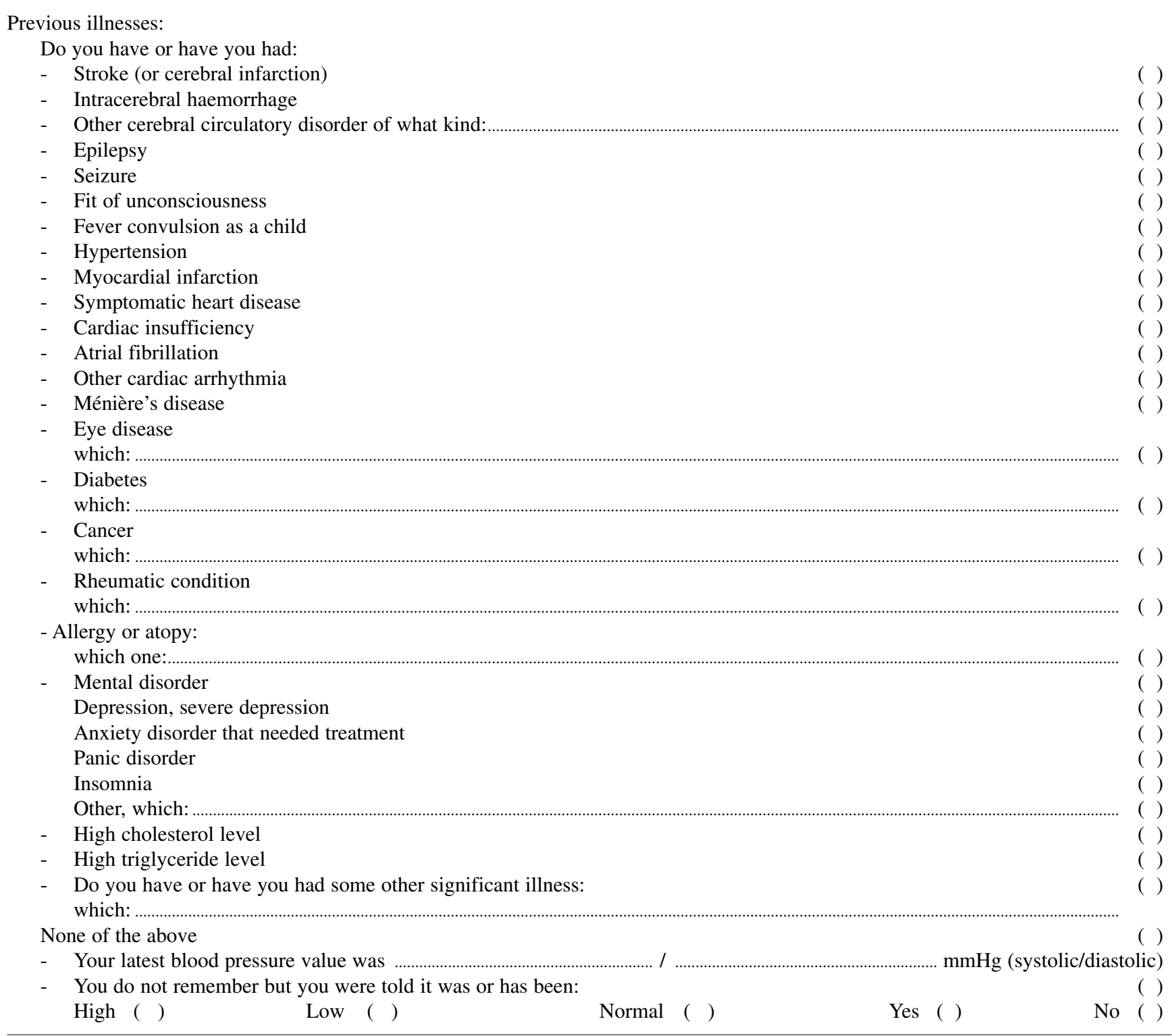

( $\mathrm{SD} \pm 18.8$ years), 40.8 years $(\mathrm{SD} \pm 18.6$ years) for women and 38.8 years $(\mathrm{SD} \pm 19.1$ years $)$ for men. Five participants, three women and two men, did not report their ages. A total of 678 were diagnosed as having migraine; 478 of these migraineurs were women and 200 men. Sixteen patients were diagnosed as Eqv, 226 individuals as MwA+MwoA, 140 individuals as MwA, 144 migraine with Unc.mig and 152 as MwoA. Mean age of the migraine patients was 38.5 years ( $\mathrm{SD} \pm 17.6$ years), 39.6 $(\mathrm{SD} \pm 17.3$ years) for women and 36.0 ( $\mathrm{SD} \pm 18.1$ years) for men. Mean age of the family members not affected by migraine was 43.1 ( $\mathrm{SD} \pm 20.8$ years), 45.4 ( $\mathrm{SD} \pm 22.3$ years) for women and 41.6 (SD \pm 19.7 years) for men.
Two participants did not report their medical illnesses and 43 did not report their blood pressure.

Distribution of comorbidities (Table 2) differed between participants with and without migraine. Migraine patients reported statistically significantly more hypotension (OR 1.43, CI 95\% 1.02-2.01), allergy (OR 1.83, CI 95\% 1.34-2.51) and psychiatric disorders (OR 4.09, CI 95\% 2.11-7.92) compared with their family members without migraine. Differences regarding epilepsy, stroke and hypertension were not statistically significant. Table 2 also shows results divided by gender.

There were 8 strokes among 304 women younger than 45 years with migraine $(2.6 \%)$. Compared to the corre- 
Table 2 Comorbidities in family members with migraine compared to family members without migraine

\begin{tabular}{lrrr}
\hline Comorbidity studied & $\begin{array}{r}\text { Family members } \\
\text { with migraine } \\
(n=676)\end{array}$ & $\begin{array}{r}\text { Family members } \\
\text { without migraine } \\
(n=322)\end{array}$ & OR (95\% CI) \\
& $\%$ & 2.9 & $1.38(0.64-2.97)$ \\
\hline Epilepsy & 4.1 & 3.4 & $0.76(0.27-2.15)$ \\
Women & 3.1 & 2.4 & $2.84(0.89-9.04)$ \\
Men & 6.1 & 1.6 & $1.74(0.66-4.60)$ \\
Stroke & 2.5 & 2.2 & $1.36(0.37-5.10)$ \\
Women & 2.5 & 1.3 & $2.39(0.48-11.73)$ \\
Men & 2.5 & 11.6 & $1.11(0.71-1.73)$ \\
Hypertension & 11.8 & 11.3 & $1.25(0.65-2.39)$ \\
Women & 11.5 & 11.5 & $1.12(0.57-2.18)$ \\
Men & 12.1 & 20.7 & $1.43(1.02-2.01)$ \\
Hypotension & 26.0 & 30.8 & $1.32(0.83-2.09)$ \\
Women & 32.1 & 16.3 & $0.64(0.32-1.14)$ \\
Men & 10.7 & 22.0 & $1.83(1.34-2.51)$ \\
Allergy & 34.0 & 22.0 & $2.01(1.26-3.19)$ \\
Women & 37.4 & 22.2 & $1.18(0.73-1.92)$ \\
Men & 25.4 & 2.9 & $4.09(2.11-7.92)$ \\
Psychiatric disorder & 11.1 & 5.6 & $2.82(1.28-6.19)$ \\
Women & 12.9 & 1.3 & $3.90(1.03-14.67)$ \\
Men & 5.1 & & \\
& & & \\
\hline
\end{tabular}

Results are controlled for age

$C I$, confidence interval; $O R$, odds ratio

Table 3 Comorbidities in women with migraine compared to men with migraine

\begin{tabular}{lrrr}
\hline Comorbidity studied & Women with migraine, \% & Men with migraine, \% & OR (95\% CI) \\
\hline Epilepsy & 3.1 & 6.1 & $0.50(0.23-1.07)$ \\
Stroke & 2.5 & 2.5 & $1.1(0.35-3.51)$ \\
Hypertension & 11.5 & 12.1 & $0.85(0.48-1.53)$ \\
Hypotension & 32.1 & 10.7 & $4.40(2.65-7.32)$ \\
Allergy & 37.4 & 25.4 & $1.91(1.31-2.78)$ \\
Psychiatric disorder & 12.9 & 5.1 & $2.67(1.34-5.34)$
\end{tabular}

Results are controlled for age

$O R$, odds ratio; $C I$, confidence interval

sponding figure in non-migraineurs ( 1 stroke in 57 participants, $1.7 \%$ ), the difference is not statistically significant.

Some gender differences were observed between migraineurs (Table 3). Women with migraine had significantly more hypotension (OR 4.40, CI 95\% 2.65-7.32), allergy (OR 1.91, CI 95\% 1.31-2.78) and psychiatric disorders (OR 2.67, CI 95\% 1.34-5.34) than men with migraine.

Table 4 presents reported comorbidities in the different diagnostic categories of the study. The MwA, MwA+MwoA and Unc.mig groups reported significantly more allergy and psychiatric disorders compared to the NO category (MwA OR 1.68 (CI 95\% 1.01-2.79) for allergy and OR 3.46 (CI 95\% 1.17-10.21) for psychiatric disorders, MwA+MwoA OR 1.86 (CI 95\% 1.17-2.97) for allergy and OR 5.91 (CI 95\% 2.16-16.20) for psychiatric disorders and Unc.mig OR 1.68 (CI 95\% 1.02-2.78) for allergy and OR 5.21 (CI 95\% 1.80-15.05) for psychiatric disorders.

Additionally, subgroup analysis showed that women with MwA+MwoA reported more hypotension (OR 2.12, CI 95\% 1.065-4.23) and men with MwA+MwoA reported more epilepsy (OR 6.76, CI 95\% 1.028-44.48) and stroke (OR 15.19, CI 95\% 1.15-200.88) than the NO group. Epilepsy was also common among men with Unc.mig (OR 5.88, CI 95\% 1.063-32.57) compared to the NO group.

There was no significant difference between MwoA and NO groups or between Eqv and NO groups. 


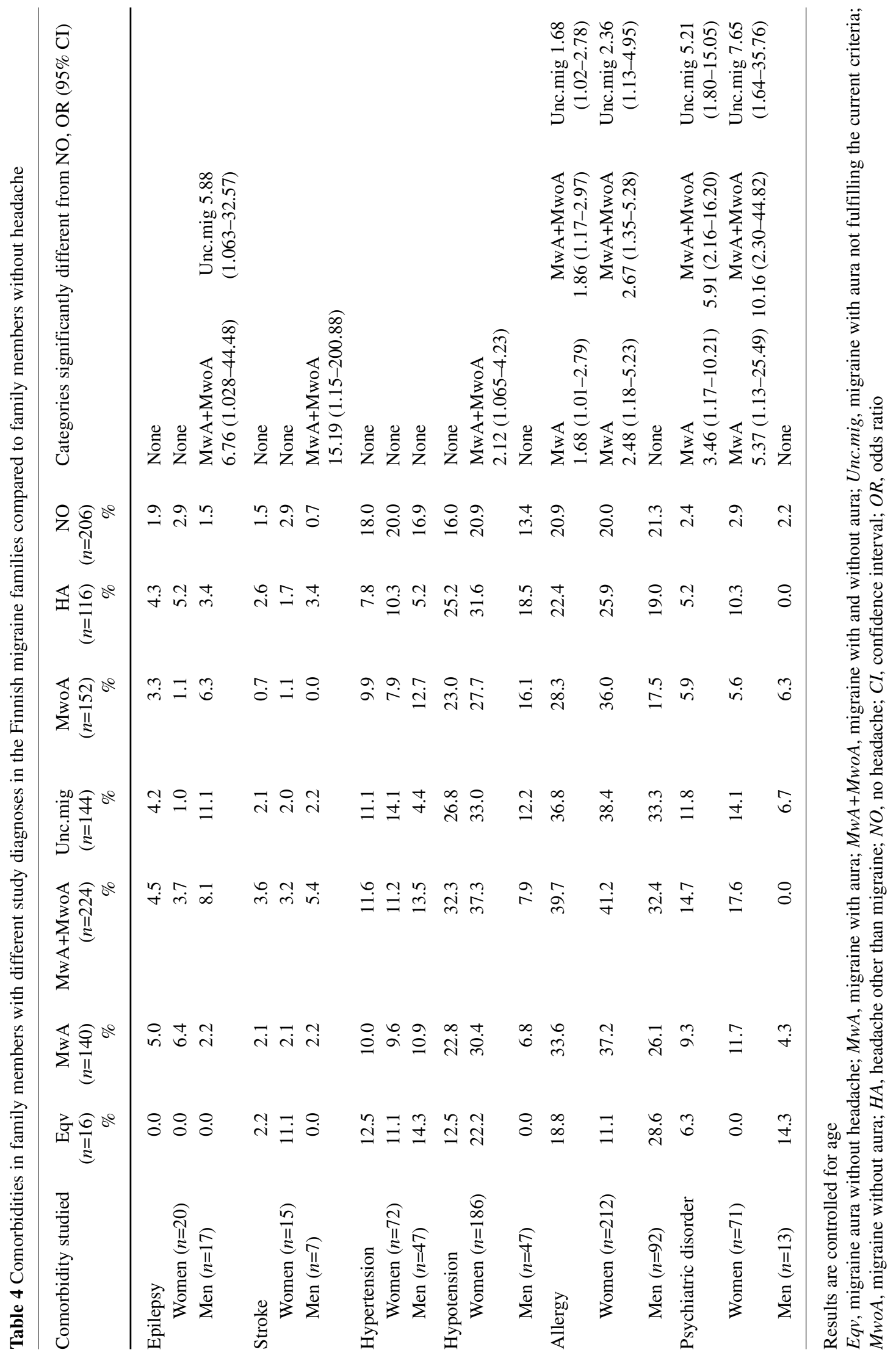




\section{Discussion}

Results of this Finnish family study confirm many previous reports concerning comorbidity of migraine $[5,6,10]$.

In the study population allergy and psychiatric conditions were comorbid with migraine, as reported previously [11-13]. In a more detailed analysis the disorders were especially prevalent among the MwA+MwoA, Unc.mig and MwA phenotypes. Thus allergy and psychiatric conditions could have more links to the aura phase of migraine. This association is clearly more pronounced among women.

A strong association of migraine and epilepsy has been shown in a previous family study by Ottman and Lipton [10]. In our families epilepsy was significantly associated with migraine only among men. Again it seems that association is somehow linked to the aura phase because statistical significance was found only in Mwa+MwoA and Unc.mig groups. Thus it seems that the common background underlying migraine and epilepsy exists also in these families, but the association is only minor, and possibly greater among men. It is clear that caution is warranted concerning the observation, because of the small number of epilepsy cases (17) among participating men. Overall it can be stated again that further studies, also genetic, are needed to elucidate the interesting links between migraine and epilepsy.

The well known association of migraine and stroke [14] was not striking. In the rare cases when stroke was present, it seemed to occur more often in patients with aura, as has been reported before [14]. The only significant finding in this regard was that men who fulfilled the criteria of both migraine with and without aura had a 15.19 times higher risk for stroke than men without migraine. Here again the small number of strokes has to be noted. Probably there are multiple, acquired and genetic, factors that occasionally cause a migraine patient to have a stroke. The familial liability to migraine seems to be a rather small contributor.

We found an association between hypotension and migraine among the female participants. The risk for hypotension was 4.4 times higher among women with migraine than men with migraine. Especially the MwA+MwoA phenotype stands out. The relationship between blood pressure and migraine is controversial. Most of the studies that show an association between hypertension and migraine were done before the introduction of the International Headache Society (IHS) criteria in 1988. Recently Wiehe et al. [15] and Lee et al. [16] reported an association between low blood pressure and migraine. Again further and more detailed studies into this association will be very interesting. It seems that gender and migraine subgroup related differences are important. All significant findings were from subgroups of patients who have aura (MwA, MwA+MwoA, Unc.mig).

Some reservations are appropriate when analysing the presented data. The applied questionnaire, the FMSQFS, has been validated for migraine, not for the comorbid diseases. Table 1 presents the wording (translated into English) concerning associated diseases in the questionnaire. Thus, precise incidence or prevalence figures cannot be drawn from the data. This might be especially notable concerning specific psychiatric disorders. The focus should be on the reported differences between those with and those without migraine. This is of course a limitation, but as the FMSQFS is already extensive, more precise questioning would probably have resulted in exhaustion of the participants. Overall, it can be said that the presented observations are very much in line with previous literature, which further supports the reliability of the methods used in the present study.

There are several implications of the data when migraine pathophysiology and treatment are considered. Prophylaxis of stroke and hypertension are important also in familial migraine, but overemphasis is not warranted. Treatment of allergic conditions is important, and could also relieve migraine. Psychiatric conditions, especially depression, are essential, and their treatment should be part of comprehensive migraine care. Antiepileptic medications could be especially good choices for migraine prophylaxis if there is a family history of comorbid epilepsy, especially among men, and if there is a tendency towards low blood pressure in the family, other prophylaxis besides beta-blockers seems appropriate. From a pathophysiological point of view, studies involving ion channels (epilepsy), mechanisms related to the ANS (hypotension), immunology (allergy) and serotonergic transmission (psychiatric conditions) are indicated.

The presented data show that family data can be effectively used to study comorbidity of migraine. Besides therapeutical implications, this can be important when the elusive genes for the common forms of migraine, migraine with and without aura, are searched for. Migraine families can be sorted out based on their familial comorbidities (epilepsy, stroke, hypertension, hypotension, allergy, psychiatric conditions) and the groups studied separately. This, most probably, could lead to more homogenous study populations and, at the same time, the likelihood that the current molecular genetic methods can locate new migraine genes is increased.

Acknowledgements This study was supported by funding from the Finnish Neurology Foundation, the Research Funds of the Helsinki University Hospital, the Academy of Finland (MW, AP) and NIH grant R01 N537675-02 (to AP). 


\section{References}

1. Ophoff RA, Terwindt GM, Vergouve MN et al (1996) 2+ channel gene CACNL1A4. Cell 87:543-552

2. Fusco MD, Marconi R, Silvestri L et al (2003) Haploinsufficiency of ATP1A2 encoding the $\mathrm{Na}+\mathrm{K}+$ pump 2 subunit associated with familial hemiplegic migraine type 2 . Nat Genet 33:192-196

3. Terwindt GM, Ophoff RA, Haan J, Sandkuijl LA (1998) Migraine, ataxia and epilepsy: a challenging spectrum of genetically determined channelopathies. Dutch Migraine Genetics Research Group. Eur J Hum Genet 6:297-307

4. Ptacek LJ (1998) The place of migraine as a channelopathy. Curr Opin Neurol 11:217-226

5. Merikangas KR, Rasmussen BK (2000) Migraine comorbidity. In: Olesen J, Tfelt-Hansen P, Welch KMA (eds) The headaches, 2nd Edn. Lippincott Williams \& Wilkins, Philadelphia, pp 235-240
6. Breslau N, Rasmussen BK (2001) The impact of migraine: epidemiology, risk factors, and co-morbidities. Neurology 56:4S-12S

7. Kallela M, Wessman M, Färkkilä M et al (2001) Familial migraine with and without aura: clinical characteristics and co-occurrence. Cephalalgia 8:441-449

8. Kallela M, Wessman M, Färkkilä M (2001) Validation of a migraine specific questionnaire for family studies. Eur J Neurol 8:61-66

9. International Headache Society Classification Subcommittee (2004) The International Classification of Headache Disorders, 2nd edition. Cephalalgia 24[Suppl 1]:9-160

10. Ottman R, Lipton RB (1994) Comorbidity of migraine and epilepsy. Neurology 44:2105-2110

11. Chen TC, Leviton A (1990) Asthma and eczema in children born to women with migraine. Arch Neurol 47:1227-1230
12. Mortimer MJ, Kay J, Grawkrodger DJ et al (1993) The prevalence of headache and migraine in atopic children: an epidemiological study in general practice. Headache 33:427-431

13. Breslau N, Davis GC, Andreski P (1991) Migraine, psychiatric disorders, and suicide attempts: en epidemiological study of young adults. Psychiatry Res 37:11-23

14. Tzourdio C, Tehindrazanarivelo A, Igleasias $\mathrm{S}$ et al (1995) Case-control study of migraine and risk of ischemic stroke in young women. Br Med J 310:830-833

15. Wiehe M, Fuchs S, Moreira L et al (2002) Migraine is more frequent in individuals with optimal and normal blood pressure: a population based study. J Hypertens 20:1303-1306

16. Lee H-C, Coulter CL, Adickes ED, et al (1996) Autonomic ganglionitis with severe hypertension, migraine, and episodic but fatal hypotension. Neurology 47:817-821 